\title{
Sleep Disorders in Kidney Diseases
}

${ }^{1}$ Rohit Kumar, ${ }^{2}$ Jagdish C Suri, ${ }^{3}$ Manas K Sen

\begin{abstract}
Sleep disorders are common, multi-factorial and often underrecognized in patients with renal diseases. Sleep disorders have a negative impact on the wellbeing and quality of life. Medical literature in the past few years has documented many definitive associations between kidney diseases and various sleep disorders. Prompt referral to a sleep medicine specialist for further evaluation is warranted in this group of patients at the earliest suspicion of an insidious sleep disorder. In this review, we will discuss the common sleep disorders seen in patients with kidney disorders-the restless leg syndrome/Willis-Ekbom disease (RLS/WED), sleep-disordered breathing (SDB), insomnia disorder, hypersomnolence disorder, circadian rhythm sleep-wake disorders (CRSWDs) and parasomnias.
\end{abstract}

Keywords: Kidney disease, Renal Failure, Sleep apnoea

How to cite this article: Kumar R, Suri JC, Sen MK. Sleep Disorders in Kidney Diseases. Indian Sleep Med 2018;13(2):29-35.

\section{Source of support: Nil}

Conflict of interest: None

\section{INTRODUCTION}

Sleep disorders have been commonly linked with kidney disease and are often diagnosed late in these patients as physicians fail to identify or are unaware of the early symptoms of these sleep disorders. ${ }^{1}$ They have also been shown to have an adverse impact on the quality of life and overall survival. ${ }^{2,3}$ In the last few decades, the associations and intricate relationships between sleep disorders and renal diseases have been realized. ${ }^{4}$ Earlier, only restless leg syndrome and obstructive sleep apnea were recognized as common disorders associated with renal diseases; however, it has been observed that patients with chronic kidney disease (CKD) appear to experience a myriad of sleep disorders. The complex and dynamic relationship between sleep disorders and CKD remains relatively less investigated.

\footnotetext{
${ }^{1}$ Resident, ${ }^{2}$ Consultant Professor, ${ }^{3}$ Professor

${ }^{1-3}$ Department of Pulmonary, Sleep, Critical Care, Vardhman Mahavir Medical College, Safdarjung Hospital, New Delhi, India

Corresponding Author: Jagdish C Suri, Consultant Professor Department of Pulmonary, Sleep and Critical Care, Vardhman Mahavir Medical College, Safdarjung Hospital, New Delhi, India, e-mail docjcsuri@gmail.com
}

\section{RESTLESS LEG SYNDROME/WILLIS-EKBOM DISEASE}

RLS/WED refers to complaints of an unpleasant sensation in the lower extremities accompanied by an uncontrollable urge to move them. ${ }^{5}$ This occurs typically in the evening or early part of the night; it is worse during periods of inactivity and transiently relieved by movement. ${ }^{6}$ The timing of the symptoms often leads to a low quality of life, insomnia, and a consequent impairment of daytime activity.

A study of 500 patients stratified according to estimated glomerular filtration rate, assessed the prevalence of RLS/WED. It estimated the prevalence of RLS to be $18.9 \%$ in patients with estimated glomerular filtration rate (eGFR) $\geq 60,26 \%$ in CKD patients, and $26 \%$ in the end-stage renal disease (ESRD) patients. ${ }^{7}$ A recent metaanalysis of 51 studies, had estimated that one-quarter of patients with CKD had RLS/WED, especially those who were on dialysis. ${ }^{8}$

The pathophysiology of RLS remains unclear. Although there are no gross structural brain abnormalities in RLS, widespread brain areas are activated, including the pre-and post-central gyri, cingulate cortex, thalamus, and cerebellum. Pathologically, the most consistent finding is a striatal iron deficiency in RLS patients. A host of other biological systems are also altered in RLS, including the dopaminergic, oxygen-sensing, opioid, glutamatergic, and serotonergic systems. ${ }^{9,10}$ In patients with renal diseases, the predominant etiology of RLS appears to be related to iron deficiency. ${ }^{11}$ Iron deficiency is common in patients with renal diseases. Blood loss during dialysis is believed to exacerbate the iron deficiency and perhaps is one of the primary reasons for the development of RLS. ${ }^{12,13}$ In addition, the patients, due to renal impairment and dialysis, often develop an imbalance in various metabolites. Animal experiments have documented changes in the level of neurotransmitters in the brain, depending on the stage of renal insufficiency. ${ }^{14}$ These changes in the level of neurotransmitters may also have a role in the pathogenesis of RLS/WED.

Treatment for RLS/WED depends on the severity and frequency of RLS symptoms, and comprises of non-pharmacological and pharmacological interventions. Good sleep hygiene can help prevent the development of insomnia related to RLS. Aerobic exercises during hemodialysis have also been shown to improve the symp- 
toms of restless leg syndrome. ${ }^{15}$ Treatment measures also include reduction of potential exacerbating factors such as excess caffeine, alcohol and nicotine, medical conditions (anemia, iron deficiency), and medications (tricyclic antidepressants, serotonin reuptake inhibitors, dopamine antagonists). If iron stores are low, iron supplementation may improve the symptoms.

Even though there are recommended pharmacological treatment guidelines for the general population, limited information is available on the effects of these therapies in ESRD patients. Medications used to treat RLS include L-dopa and dopamine agonists such as pramipexole, but this often fails to fully resolve RLS. ${ }^{16}$ Patients may experience augmentation, an increase in RLS symptom severity with increasing drug dosage, which is the main complication of dopaminergic drugs. Gabapentin may also be considered as an alternative medication. The effectiveness of benzodiazepines for RLS treatment is currently unknown. ${ }^{17}$ The definitive treatment of any disease requires resolution of the underlying etiology. Renal diseases are often progressive and normal kidney function is unlikely to be restored medically. Increasing the dose of dialysis ${ }^{18}$ and renal transplantation ${ }^{19,20}$ has been shown to ameliorate the symptoms of RLS/ WED.

\section{SLEEP-RELATED BREATHING DISORDERS/ SLEEP DISORDERED BREATHING}

Sleep disordered breathing (SDB) describes a group of disorders characterized by an abnormal respiratory pattern or insufficient ventilation during sleep. These include broadly two types of apneas-the obstructive and the central sleep apneas.

\section{Obstructive Sleep Apnea}

Obstructive Sleep Apnea (OSA) comprises of apneas (complete or near complete cessation of airflow), hypopneas (partial airway obstruction), or respiratory effortrelated arousals (RERAs-limited airway obstruction with an associated arousal). Sleep-disordered breathing disrupts the patient's sleep pattern, which not only makes the patient tired and exhausted the next day but, may also put excessive strain on their nervous system and major organs. The coexistence of renal disease and OSA can cause an exaggerated acidosis (because of a combined metabolic and respiratory acidosis) beyond what would be expected in a patient with either disease alone.

It has been observed that patients with OSA are at a higher risk of acute kidney injury during critical illness. ${ }^{21}$ In a longitudinal population-based cohort study, patients with OSA even without diabetes or hypertension are at higher risk of developing CKD over the next three years than the non-OSA cohort. ${ }^{22}$ Hypoxia has been postulated to drive the pathogenesis of CKD, and this may be the dominant mechanism in such patients. ${ }^{23}$

Also, the prevalence of OSA is higher in patients with renal disease. While prevalence in the general population is estimated to be $9-24 \%,{ }^{24}$ the prevalence of OSA in ESRD patients is estimated to be around $34-51 \%$, through selfreport questionnaires, ${ }^{25-27}$ and about $24-66 \%$ when based on polysomnography. ${ }^{28,29}$

Several studies have examined "rostral fluid shift" as a possible mechanism in the pathogenesis of OSA in CKD patients. Patients with kidney disease accumulate fluid throughout their body, and when they lie down to sleep, there is a migration of the fluid to their upper airway, in a phenomenon termed as rostral fluid shifting. ${ }^{30,31}$ It has also been seen that as renal function deteriorates, the apnea-hypopnea index (AHI) can increase.

The treatment of OSA in renal disease is similar to the general population; with continuous positive airway pressure (CPAP) as the first line of treatment. Other treatment modalities in the general population include the use of dental appliances, oral surgery, and treating comorbid medical conditions like obesity or hypothyroidism. ${ }^{32}$ However, these modalities have not yet been extensively studied in the CKD population.

The use of methods that can reduce the accumulated fluid may help to improve OSA. The use of nocturnal hemodialysis has been shown to improve the pharyngeal size in renal patients with OSA. ${ }^{33}$ Similarly, in patients with nephrotic syndrome who can have nocturnal rostral fluid shifting, treatment with steroids can improve the OSA. ${ }^{34}$ Renal Transplantation has also been shown to ameliorate OSA in such patients. ${ }^{35}$

\section{Central Sleep Apnea}

Central sleep apnoea (CSA) is characterized by recurrent apnoeic episodes with no associated respiratory effort. CSA in adults is defined as a cessation in the airflow of ten or more seconds in the absence of any inspiratory effort. CSA is diagnosed when $50 \%$ or more of the events are central. $^{36}$

While OSA is the most common form of sleep apnea in patients with kidney disease, CSA may be under-reported as it can only be diagnosed with polysomnography tests. A systematic review reported the aggregate point prevalence of CSA in CKD is $9.6 \%, 37$ and when selecting only those studies that reported total CSA index (i.e., including both central apnea and central hypopnea indices) the prevalence was estimated to be $33.3 \% .{ }^{38}$ It has been observed that the number of CSA per hour are more in patients with worse renal function and that even after adjustment for cardiovascular co-morbidi- 
ties, kidney disease is independently associated with CSA. ${ }^{39}$

Several pathophysiological pathways are believed to contribute to the development of CSA in patients with renal disease. Some of the reported mechanisms include the overstimulation of the pulmonary receptors due to the interstitial fluid (similar to patients with congestive heart failure), chronic metabolic acidosis (causing compensatory hyperventilation and hypocapnia), severe anemia (leading to hypoxia-induced periodic breathing), decreased clearance of uremic toxins (with uremic autonomic neuropathy) and exaggerated chemo-reflex sensitivity leading to an unstable ventilatory control. ${ }^{40}$ CSA has been found to be an independent risk factor for all-cause mortality ${ }^{2}$ and hence its identification and treatment are important goals in the management of such patients. Apart from the conventional treatment of CSA, nocturnal hemodialysis and kidney transplantation have been shown to reduce the central apneas in such patients. ${ }^{41}$

\section{Insomnia Disorder}

Insomnia was earlier merely described as a lack of sleep and in the previous classification (DSM IV) had been classified as primary or secondary. ${ }^{42}$ However, the differentiation between primary and secondary insomnia was proved more hypothetical than actual and based upon the current evidence insomnia subtypes described in the earlier system have been lumped into one-insomnia disorder. Research in this field suggests that insomnia occurring during medical disorders has a bidirectional and interactive relationship with the coexisting medical illnesses. ${ }^{43}$ Insomnia has been recognized as a distinct disorder rather than just a symptom, and diagnosis is assigned to individuals who experience recurrent poor sleep quality or quantity that causes distress or impairment in important areas of functioning. ${ }^{44}$ Insomnia as a disorder needs independent clinical attention.

Insomnia is a common symptom in the general population (prevalence ranges from 5 to $19 \%$ in different studies $)^{45}$ and is significantly more common in renal patients (approximately 45-70\% of ESRD patients experience symptoms of insomnia). ${ }^{46,47}$ Studies evaluating insomnia among patients with varying severity of renal dysfunction have observed that with declining renal function, there is a worsening of the sleep quality and quantity. $28,48,49$

The pathogenesis of insomnia in such patients is often multifactorial. Patients with renal diseases suffer from a high degree of anxiety, physical stress, and chronic pain; all of which cause insomnia. It has also been seen that the dialysis shift time may have an impact. ${ }^{46}$ Furthermore, renal diseases are known to cause disturbances in normal homeostasis; the concentration of neuro-peptides and electrolytes are known to be affected. This dysregulation in homeostasis might impact sleep in various ways. ${ }^{50,51} \mathrm{It}$ has been seen that in patients with CKD, the serum levels of orexin levels (a neuropeptide associated with arousal) are increased..$^{52}$ It has been suggested that this increased level may lead to increased periods of wakefulness that can be associated with insomnia. ${ }^{53}$ Kidney dysfunction is also known to cause hyperparathyroidism and hypocalcemia. In patients with severe hyperparathyroidism on maintenance hemodialysis, when parathyroidectomy was done, it was observed that there was a resolution of the hypocalcemia and hyperphosphatemia along with amelioration in insomnia. ${ }^{54}$ The exact mechanism by which this effect occurs is not known; it is possible that the hypocalcemia is indirectly implicated in a signaling pathway that affects sleep.

Patients with insomnia should initially be screened for sleep apnea; and if detected, this should be addressed first. Treatment of insomnia includes a combination of pharmacological and non-pharmacological means. The non-pharmacological techniques include cognitive behavioral therapy (CBT) ${ }^{55}$ acupressure, ${ }^{56}$ relaxation training, ${ }^{57}$ and physical exercise. A recent systematic review has shown that in dialysis-dependent patients, CBT could shorten sleep latency, alleviate sleep disturbance and reduce the use of sleep medications. ${ }^{58}$ The core tenets of CBT are stimulus control, sleep restriction, and sleep hygiene. It is also beneficial to simultaneously treat or mitigate the other co-existing conditions, such as pain or depression, which also contribute to insomnia.

Sedative antidepressants and anxiolytics are effective in individuals who suffer from depression, worry, and insomnia, however there is little research supporting their safety and efficacy in ESRD patients. Considering the polypharmacy and altered metabolism in this patient population, treatment trials are clearly needed before they can be liberally used.

\section{Hypersomnolence Disorder}

Excessive daytime somnolence (EDS) is defined as the inability to stay awake or alert throughout the course of the day, resulting in sleepiness or inadvertent dozing during passive (reading, watching television) or active (driving, conversation) daily activity. The words sleepiness or hypersomnolence usually used to imply the abnormal tendency to go to sleep; whilst, by hypersomnia, an increased amount of sleep over a 24-hour period of time is assumed. ${ }^{59}$ EDS is frequently associated with sleep-related breathing disorders, circadian rhythm disorders and other causes of disturbed nocturnal sleep. Only 
when these conditions have been excluded, can a diagnosis of a central disorder of hypersomnolence be made. Daytime sleepiness is common in kidney patients. The prevalence of EDS in patients with renal disease varies between 44 and $67 \%$ in the reported medical literature. ${ }^{27,60}$ In a study of in patients with end-stage renal disease on hemodialysis, daytime sleepiness was assessed by the multiple sleep latency test (MSLT); the majority (54\%) of patients were pathologically sleepy with a mean sleep latency of less than five minutes. ${ }^{61}$ In another study of stable hemodialysis patients, one third had MSLT scores suggesting abnormal levels of physiological daytime sleepiness, and $13 \%$ subjects had scores consistent with severe, pathological sleepiness. Thirty percent had significant subjective daytime sleepiness as measured by the ESS. ${ }^{62}$ However, the exact prevalence of central disorders of hypersomnolence (such as narcolepsy and idiopathic hypersomnia) is not well reported in patients with renal disease. These are relatively rare diseases in the general population. A study had reported a prevalence of possible narcolepsy in dialysis-dependent patients to be about $1.4 \%$ and in patients, not on dialysis it was estimated to be about $1 \%{ }^{28}$; which is much higher than that of the general population.

The pathogenesis of hypersomnia in renal disease is likely multi-factorial; contributed by multiple factors including high prevalence of sleep disorders like SDB and RLS. ${ }^{27,28}$ Such patients also often have psychiatric co-morbidities which may also manifest as EDS. ${ }^{63}$ The other factors which are also believed to contribute to the pathogenesis are uremic encephalopathy, rapid fluid shift and $\mathrm{pH}$ changes, electrolyte imbalance, and altered levels of inflammatory cytokines and high daytime melatonin levels. It has been seen that patients undergoing hemodialysis experience ventilatory instability, fewer sleep cycles, shorter sleeping times, a predominance of light sleep and reduction in Rapid Eye Movement (REM) sleep with a consequently decreased sleep efficiency, which further aggravates the problem of hypersomnia in such patients. ${ }^{64}$

EDS can cause negative behavioral, physiologic, and cognitive effects, which limit patients' function and quality of life. ${ }^{65}$ Successful treatment of sleepiness can lead to overall improvement in the quality of life and prevent significant morbidity. The approach to the assessment of EDS in kidney patients is generally the same as in general population; requiring a detailed history and examination, along with screening for co-morbidities and occasionally requires a polysomnography followed by an MSLT. An effective treatment of the underlying cause can often reduce the EDS. An additional intervention is to switch to nocturnal hemodialysis. ${ }^{61}$ It has also been seen that nocturnal sleep quality improved after transplantation and patients reported improved quality of life and daytime sleepiness scores. ${ }^{66}$ Although there are multiple pharmacological treatments approved for narcolepsy, there are none for the other hypersomnias, and off-label use of these medications is inadequate to alleviate symptoms in most patients. ${ }^{66}$

\section{Circadian Rhythm Sleep-Wake Disorders}

The CRSWDs encompass a wide spectrum of diseases which primarily arise due to the pathology of the circadian clock or to a misalignment of the timing of the endogenous circadian rhythm with the environment. ${ }^{67}$ The DSM-5 classification recognizes five subtypes of CRSWD: delayed sleep phase, advanced sleep phase, non-24-hour sleep-wake, irregular sleep-wake, and shift work types. Of these, the delayed sleep phase and the advanced sleep phase are the common subtypes seen in clinical practice. There is scarce information regarding CRSWDs in patients with renal disease. Actigraphy studies have rarely been performed in kidney patients.

The kidney patients are believed to be at risk for these ailments primarily because of derangements in the melatonin secretion. Melatonin, a hormone secreted by the pineal gland, is responsible for the sleep-wake circadian rhythm. It is secreted in small amounts during daytime but increases during the night, a phenomenon called dim light melatonin onset (DLMO), which correlates with the onset of nocturnal sleepiness. It has been seen that the melatonin amplitude and melatonin rhythm decreases with advancing renal dysfunction. ${ }^{68}$ The high level of tumor neurosis factor (TNF), seen in patients with renal diseases, is probably involved in the blockage of melatonin production. In addition, it has been observed that the circadian melatonin rhythm may be absent in ambulatory peritoneal dialysis and conventional daytime patients on HD and it re-establishes itself in patients on nocturnal hemodialysis. ${ }^{69}$ Another possible reason for the sleepwake disturbance is the frequent nocturia in these patients due to the poor renal tubular concentrating ability.

Treatment of this disorder is possible using various approaches which have the potential to resynchronize a disrupted circadian sleep-wake rhythm. These include nocturnal dialysis, lowering of dialysate temperature, administration of exogenous melatonin, administration of exogenous erythropoietin, bright light and intra-dialytic exercise. $^{70}$

\section{Parasomnias}

Parasomnias are unpleasant or undesirable behaviors or experiences that occur predominantly during or within close proximity to sleep. ${ }^{71}$ They manifest because of a perturbed arousal mechanism-there is an activation of 
the motor component though electroencephalographic electrical activity shows ongoing sleep. Parasomnias are typically classified by the sleep state from which they arise: non-rapid eye movement sleep (NREM) and REM. ${ }^{72}$

The prevalence of parasomnia in renal disease has not been extensively studied and it is difficult to estimate its true prevalence. In a study of patients with end-stage renal disease on hemodialysis, it was seen that $13 \%$ of the patients had nightmares, $2 \%$ had sleepwalking, and $2 \%$ had possible REM sleep behavior disorder (RBD); while amongst CKD patients (not on hemodialysis) 15\% of the patients had nightmares, $4 \%$ had sleepwalking and 3\% had RBD. ${ }^{28}$ This is slightly higher than the prevalence reported in the general population. ${ }^{73}$

Similar to the general population, high clinical suspicion is required for the identification of RBD in patients with renal disease. Studies specifically evaluating treatment of parasomnia in renal disease are lacking and currently are treated using behavioral and cognitivebehavioral managements; ${ }^{74}$ only occasionally resorting to pharmacotherapy.

\section{CONCLUSION}

There is a need for the nephrologists and the sleep specialist to jointly recognize and tackle the sleep complaints in patients with renal disease. In such patients, the identification, diagnosis, and treatment of sleep disorders are complicated by the overlapping presentation of renal and sleep disorders with other common co-morbid conditions. The treatment of sleep disorders should be multi-layered and comprehensive. Future research should attempt to explain the complex interrelationships between sleep and kidney disease, test standard treatments in these specific patient groups and develop novel treatments for sleep disorders that can address the complex psychosocial and pathophysiological processes in these patients.

\section{REFERENCES}

1. Pierratos A, Hanly PJ. Sleep disorders over the full range of chronic kidney disease. Blood Purif. 2011;31(1-3):146-150.

2. Xu J, Yoon IY, Chin HJ. The effect of sleep apnea on all-cause mortality in nondialyzed chronic kidney disease patients. Sleep Med. 2016 Nov-Dec;27-28:32-38.

3. Parvan K, lakdizaji S, Roshangar F, Mostofi M. Quality of Sleep and its Relationship to Quality of Life in Hemodialysis Patients. Journal of Caring Sciences. 2013 Dec;2(4):295-304.

4. Maung SC, El Sara A, Chapman C, Cohen D, Cukor D. Sleep disorders and chronic kidney disease. World J Nephrol. 2016 May 6;5(3):224-232.

5. Klingelhoefer L, Bhattacharya K, Reichmann H. Restless legs syndrome. Clin Med (Lond). 2016 Aug;16(4):379-382.

6. Rinaldi F, Galbiati A, Marelli S, Cusmai M, Gasperi A, Oldani A, Zucconi M, Padovani A, Strambi LE. Defining the phenotype of restless legs syndrome/Willis-Ekbom disease (RLS/WED): a clinical and polysomnographic study. J Neurol. 2016 Jan 2;263(2):396-402.

7. Lee J, Nicholl DDM, Ahmed SB, Loewen AHS, Hemmelgarn BR, Beecroft JM, Turin TC, Hanly PJ. The Prevalence of Restless Legs Syndrome across the Full Spectrum of Kidney Disease. Journal of Clinical Sleep Medicine : JCSM : Official Publication of the American Academy of Sleep Medicine. 2013 May 15;9(5):455-459.

8. Lin Z, Zhao C, Luo Q, Xia X, Yu X, Huang F. Prevalence of restless legs syndrome in chronic kidney disease: a systematic review and meta-analysis of observational studies. Ren Fail. 2016 Oct 20;38(9):1335-1346.

9. Koo BB, Bagai K, Walters AS. Restless Legs Syndrome: Current Concepts about Disease Pathophysiology. Tremor Other Hyperkinet Mov (N Y). 2016 Jul;6:401.

10. Allen RP. Restless Leg Syndrome/Willis-Ekbom Disease Pathophysiology. Sleep Medicine Clinics. 2015 Sep;10(3):207214.

11. Gigli GL, Adorati M, Dolso P, Piani A, Valente M, Brotini $\mathrm{S}$. Restless legs syndrome in end-stage renal disease. Sleep Med. 2004 May;5(3):309-315.

12. Kavanagh D, Siddiqui S, Geddes CC. Restless legs syndrome in patients on dialysis. Am J Kidney Dis. 2004 May 1;43(5):763-771.

13. Beladi-Mousavi SS, Jafarizade $M$, Shayanpour S, Bahadoram M, Moosavian SM, Houshmand G. Restless Legs Syndrome: Associated Risk Factors in Hemodialysis Patients. Nephrourol Mon. 2015 Nov 29;7(6):e31967.

14. Schmid G, Bahner U, Peschkes J, Heidland A. Neurotransmitter and monoaminergic amino acid precursor levels in rat brain: effects of chronic renal failure and of malnutrition. Miner Electrolyte Metab. 1996;22(1-3):115-118.

15. Mortazavi M, Vahdatpour B, Ghasempour A, Taheri D, Shahidi S, Moeinzadeh F, Dolatkhah B, Dolatkhah S. Aerobic exercise improves signs of restless leg syndrome in end stage renal disease patients suffering chronic hemodialysis. ScientificWorldJournal. 2013 Nov 6;2013:628142.

16. Allen RP, Picchietti D, Hening WA, Trenkwalder C, Walters AS, Montplaisi J. Restless legs syndrome: diagnostic criteria, special considerations, and epidemiology. A report from the restless legs syndrome diagnosis and epidemiology workshop at the National Institutes of Health. Sleep Med. 2003 Mar;4(2):101-119.

17. Carlos K, Prado GF, Teixeira CD, Conti C, de Oliveira MM, Prado LB, et al. Benzodiazepines for restless legs syndrome. Cochrane Database Syst Rev. 2017 Mar 20;3:CD006939.

18. Jaber BL, Schiller B, Burkart JM, Daoui R, Kraus MA, Lee Y, Milar BM, Teitel Baum I, Williams AW, Finkelstei FO, Impact of short daily hemodialysis on restless legs symptoms and sleep disturbances. Clin J Am Soc Nephrol. 2011 May;6(5):1049-1056.

19. Azar SA, Hatefi R, Talebi M. Evaluation of effect of renal transplantation in treatment of restless legs syndrome. Transplant Proc. 2007 May;39(4):1132-1133.

20. Winkelmann J, Stautner A, Samtleben W, Trenkwalder C. Long-term course of restless legs syndrome in dialysis patients after kidney transplantation. Mov Disord. 2002 Sep;17(5):1072-1076.

21. Dou L, Lan H, Reynolds DJ, Gunderson TM, Kashyap R, Gajic O, Caples S, Li G, Kashani JB. Association between Obstructive Sleep Apnea and Acute Kidney Injury in Criti- 
cally Ill Patients: A Propensity-Matched Study. Nephron. 2017;135(2):137-146.

22. Lin YS, Liu PH, Lin SW, Chuang LP, Ho WJ, Chou YT, et al. Simple obstructive sleep apnea patients without hypertension or diabetes accelerate kidney dysfunction: a population follow-up cohort study from Taiwan. Sleep Breath. 2017 Mar;21(1):85-91.

23. Fu Q, Colgan SP, Shelley CS. Hypoxia: The Force that Drives Chronic Kidney Disease. Clin Med Res. 2016 Mar;14(1):1539.

24. Young T, Palta M, Dempsey J, Skatrud J, Weber S, Badr $\mathrm{S}$. The occurrence of sleep-disordered breathing among middle-aged adults. N Engl J Med. 1993 Apr 29;328(17):12301235.

25. Wali SO, Alkhouli A, Howladar M, Ahmad I, Alshohaib S, Al-Ghamdi S, Kayem A. Risk of obstructive sleep apnea among Saudis with chronic renal failure on hemodialysis. Ann Thorac Med. 2015 Oct-Dec;10(4):263-268.

26. Nicholl DD, Ahmed SB, Loewen AH, Hemmelgarn BR, Sola DY, Beecroft JM, Turin TC, Henly PJ. Diagnostic value of screening instruments for identifying obstructive sleep apnea in kidney failure. J Clin Sleep Med. 2013 Jan 15;9(1):3138.

27. Al-Jahdali H. Prevalence of sleep apnea and excessive day time sleepiness in patients with end-stage renal disease on dialysis. Saudi J Kidney Dis Transpl. 2012 Mar 1;23(2):251261.

28. Ezzat H, Mohab A. Prevalence of sleep disorders among ESRD patients. Ren Fail. 2015 Jul 3;37(6):1013-1019.

29. Huang HC, Walters G, Talaulikar G, Figurski D, Carroll A, Hurwitz M, Karte K, Singer R. Sleep apnea prevalence in chronic kidney disease - association with total body water and symptoms. BMC Nephrol. 2017 Apr 4;18(1):125.

30. Elias RM, Bradley TD, Kasai T, Motwani SS, Chan CT. Rostral overnight fluid shift in end-stage renal disease: relationship with obstructive sleep apnea. Nephrology Dialysis Transplantation. 2011 Nov 5;27(4):1569-73.

31. Elias RM, Chan CT, Paul N, Motwani SS, Kasai T, Gabriel JM, Spiller N, Bradely TD. Relationship of pharyngeal water content and jugular volume with severity of obstructive sleep apnea in renal failure. Nephrol Dial Transplant. 2013 Apr;28(4):937-944.

32. Spicuzza L, Caruso D, Di Maria G. Obstructive sleep apnoea syndrome and its management. Therapeutic Advances in Chronic Disease. 2015 Sep;6(5):273-285.

33. Beecroft JM, Hoffstein V, Pierratos A, Chan CT, McFarlane P, Hanly PJ. Nocturnal haemodialysis increases pharyngeal size in patients with sleep apnoea and end-stage renal disease. Nephrol Dial Transplant. 2008 Feb;23(2):673679.

34. Tang SC, Lam B, Lam JC, Chan CK, Chow CC, Ho YW, Ip MS, Lai KN. Impact of nephrotic edema of the lower limbs on obstructive sleep apnea: gathering a unifying concept for the pathogenetic role of nocturnal rostral fluid shift. Nephrology Dialysis Transplantation. 2012 Jan 16;27(7):2788-94.

35. Lee JJ, Kim GS, Kim JA, Kim SJ, Kang JG, Kim GH, Jung HH. Improvement of sleep-related breathing disorder in patients with end-stage renal disease after kidney transplantation. Clin Transplant. 2011 Jan-Feb;25(1):126-130.

36. Muza RT. Central sleep apnoea-a clinical review. Journal of Thoracic Disease. 2015 May;7(5):930-937.
37. Nigam G, Pathak C, Riaz M. A systematic review of central sleep apnea in adult patients with chronic kidney disease. Sleep Breath. 2016 Sep 1;20(3):957-964.

38. Nigam G, Riaz M. Probing the prevalence of central sleep apnea in patients with chronic kidney disease. Sleep Breath. 2016 Dec 1;20(4):1275-1276.

39. Fleischmann G, Fillafer G, Matterer H, Skrabal F, Kotanko P. Prevalence of chronic kidney disease in patients with suspected sleep apnoea. Nephrol Dial Transplant. 2010 Jan;25(1):181-186.

40. Nigam G, Riaz M. Pathophysiology of central sleep apnea in chronic kidney disease. Saudi J Kidney Dis Transpl. 2016 Sep 1;27(5):1068-70.

41. Hanly PJ, Pierratos A. Improvement of sleep apnea in patients with chronic renal failure who undergo nocturnal hemodialysis. N Engl J Med. 2001 Jan 11;344(2):102-107.

42. Diagnostic and statistical manual of mental disorders : DSM-IV: Fourth edition. Washington, DC : American Psychiatric Association, 1994.

43. Gupta R, Zalai D, Spence DW, BaHammam AS, Ramasubramanian C, Monti JM, Pandi-Perumal SR. When insomnia is not just insomnia: the deeper correlates of disturbed sleep with reference to DSM-5. Asian J Psychiatr. 2014 Dec;12:2330.

44. Diagnostic and statistical manual of mental disorders : DSM-5: Fifth edition. Arlington, VA : American Psychiatric Publishing, 2013.

45. Riemann D, Baglioni C, Bassetti C, Bjorvatn B, Dolenc Groselj L, Ellis JG, Espie CA, Garcia-Borreguero A, Gjerstad $\mathrm{M}$, Gonclaves et al. European guideline for the diagnosis and treatment of insomnia. J Sleep Res. 2017 Dec;26(6):675700.

46. Sabbatini M, Minale B, Crispo A, Pisani A, Ragosta A, Esposito R, et al. Insomnia in maintenance haemodialysis patients. Nephrol Dial Transplant. 2002 May 1;17(5):852-856.

47. Merlino G, Piani A, Dolso P, Adorati M, Cancelli I, Valente M, et al. Sleep disorders in patients with end-stage renal disease undergoing dialysis therapy. Nephrol Dial Transplant. 2006 Jan;21(1):184-190.

48. Ibrahim JM, Wegdan OM. Epidemiology of sleep disorders in patients with chronic renal disease in Cairo, Egypt. J Egypt Public Health Assoc. 2011 Aug;86(3-4):68-72.

49. Lin HY, Hung CC, Chang YH, Lin MY, Yang MY, Liang SS, Liu W, Chen HC, Hwang SJ. Nonapnea Sleep Disorders in Patients Younger than 65 Years Are Significantly Associated with CKD: A Nationwide Population-Based Study. PLoS One. 2015 Oct 14;10(10):e0140401.

50. Kosmadakis GC, Medcalf JF. Sleep disorders in dialysis patients. Int J Artif Organs. 2008 Nov;31(11):919-927.

51. Knezevic MZ, Djordjevic VV, Jankovic SM, Djordjevic $\mathrm{VM}$. Influence of dialysis modality and membrane flux on insomnia severity in haemodialysis patients. Nephrology (Carlton). 2013 Nov;18(11):706-711.

52. Sugimoto T, Nagake $Y$, Sugimoto $S$, Akagi S, Ichikawa H, Nakamura Y, Ogawar N, Makio H. Plasma Orexin Concentrations in Patients on Hemodialysis. Nephron. 2002;90(4):379-383.

53. Rayner HC. Orexin as a possible cause of insomnia in dialysis patients. Am J Kidney Dis. 2003 Jun 1;41(6):1335-6; author reply 6.

54. Esposito MG, Cesare CM, De Santo RM, Cice G, Perna AF, Violetti E, Conzo G, Bilancio G, Celci S, Annunziata 
F et al. Parathyroidectomy improves the quality of sleep in maintenance hemodialysis patients with severe hyperparathyroidism. J Nephrol. 2008 Mar-Apr;21 Suppl 13: S92-S96.

55. Chen HY, Chiang CK, Wang HH, Hung KY, Lee YJ, Peng YS, Wu KD, Tasi TJ. Cognitive-behavioral therapy for sleep disturbance in patients undergoing peritoneal dialysis: a pilot randomized controlled trial. Am J Kidney Dis. 2008 Aug;52(2):314-323.

56. Shariati A, Jahani S, Hooshmand M, Khalili N. The effect of acupressure on sleep quality in hemodialysis patients. Complement Ther Med. 2012 Dec 1;20(6):417-423.

57. Rambod M, Pourali-Mohammadi N, Pasyar N, Rafii F, Sharif F. The effect of Benson's relaxation technique on the quality of sleep of Iranian hemodialysis patients: a randomized trial. Complement Ther Med. 2013 Dec 1;21(6):577-584.

58. Yang B, Xu J, Xue Q, Wei T, Ye C, Mei C, Mao Z. Nonpharmacological interventions for improving sleep quality in patients on dialysis: systematic review and meta-analysis. Sleep Med Rev. 2015 Oct;23:68-82.

59. Berkowski JA, Shelgikar AV. Disorders of Excessive Daytime Sleepiness Including Narcolepsy and Idiopathic Hypersomnia. Sleep Med Clin. 2016 Sep 1;11(3):365-378.

60. Walker S, Fine A, Kryger MH. Sleep complaints are common in a dialysis unit. Am J Kidney Dis. 1995 Nov 1;26(5):751-756.

61. Hanly PJ, Gabor JY, Chan C, Pierratos A. Daytime sleepiness in patients with CRF: impact of nocturnal hemodialysis. Am J Kidney Dis. 2003 Feb;41(2):403-410.

62. Parker KP, Bliwise DL, Bailey JL, Rye DB. Daytime sleepiness in stable hemodialysis patients. Am J Kidney Dis. $2003 \mathrm{Feb}$ 1;41(2):394-402.

63. Teles F, Azevedo VF, Miranda CT, Miranda MP, Teixeira Mdo C, Elias RM. Depression in hemodialysis patients: the role of dialysis shift. Clinics (Sao Paulo). 2014 Mar;69(3):198-202.

64. Jurado-Gamez B, Martin-Malo A, Alvarez-Lara MA, Munoz L, Cosano A, Aljama P. Sleep disorders are underdiagnosed in patients on maintenance hemodialysis. Nephron Clin Pract. 2007;105(1):c35-c42.

65. Roth T. Effects of excessive daytime sleepiness and fatigue on overall health and cognitive function. J Clin Psychiatry. 2015 Sep 23;76(9):e1145.

66. Russcher M, Nagtegaal JE, Nurmohamed SA, Koch BC, van der Westerlaken MM, van Someren EJ, Baker SJ, Ter Wee PN, Gaillard CA. The effects of kidney transplantation on sleep, melatonin, circadian rhythm and quality of life in kidney transplant recipients and living donors. Nephron. 2015;129(1):6-15.

67. Abbott SM, Reid KJ, Zee PC. Circadian Rhythm Sleep-Wake Disorders. Psychiatr Clin North Am. 2015 Dec 1;38(4):805823.

68. Koch BC, van der Putten K, Van Someren EJ, Wielders JP, Ter Wee PM, Nagtegaal JE, Gaillard CA. Impairment of endogenous melatonin rhythm is related to the degree of chronic kidney disease (CREAM study). Nephrol Dial Transplant. 2010 Feb;25(2):513-519.

69. Koch BC, Nagtegaal JE, Hagen EC, Wee PM, Kerkhof GA. Different melatonin rhythms and sleep-wake rhythms in patients on peritoneal dialysis, daytime hemodialysis and nocturnal hemodialysis. Sleep Med. 2010 Mar 1;11(3):242-246.

70. Koch BC, Nagtegaal JE, Kerkhof GA, Ter Wee PM. Circadian sleep-wake rhythm disturbances in end-stage renal disease. Nat Rev Nephrol. 2009 Jul;5(7):407-416.

71. Fleetham JA, Fleming JAE. Parasomnias. CMAJ : Canadian Medical Association Journal. 2014 May 5;186(8):E273-E280.

72. Howell MJ. Parasomnias: An Updated Review. Neurotherapeutics. 2012 Oct 1;9(4):753-775.

73. Bjorvatn B, Gronli J, Pallesen S. Prevalence of different parasomnias in the general population. Sleep Med. 2010 Dec 1;11(10):1031-1034.

74. Galbiati A, Rinaldi F, Giora E, Ferini-Strambi L, Marelli S. Behavioural and Cognitive-Behavioural Treatments of Parasomnias. Behav Neurol. 2015;2015:786928. 